\title{
Dermatomiositis refractaria asociada a neumonía en organización tratada con rituximab. Reporte de un caso
}

\author{
Jorge Yáñez $V^{1}$, Marcela Cisternas M² $^{2}$ Velia Saldías $H^{a}$, \\ Fernando Saldías $\mathbf{P}^{1}$. \\ Refractory dermatomyositis \\ associated with chronic organizing \\ pneumonia treated with rituximab. \\ Report of one case
}

Chronic organizing pneumonia (COP) has often been reported as a pulmonary manifestation of collagen vascular diseases, mainly rheumatoid arthritis, but the association of COP and dermatomyositis (DM) has rarely been documented. We report a 55 year-old woman with well-documented DM and a COP. She was refractory to steroids and two other immunosuppressive agents therapy (cyclophosphamide and azathioprine). Therefore, rituximab ( $2 \times 1 \mathrm{~g}$ infusions) was used for treatment. During the following weeks her strength gradually increased while creatine kinase (CK), C reactive protein and erythrocyte sedimentation rate normalized. After 6 months, she had a relapse with increased muscle enzymes, fever and moderate muscle weakness. After a second course of rituximab $(2 \times 1 \mathrm{~g}$ infusions), the patient demonstrated a remarkable clinical response as indicated by an increase in muscle strength and moderate decline in creatine kinase levels. Lung abnormalities resolved significantly on high resolution chest CT scan. Thus, B-cell depletion therapy with rituximab used alone or in combination with other immunosuppressants may be a viable option in patients with polymyositis-dermatomyositis and pneumonia refractory to current therapies (Rev Méd Chile 2008; 136: 88-93).

(Key words: Dermatomyositis; Pneumonia; Rituximab)

Recibido el 7 de enero, 2008. Aceptado el 13 de mayo, 2008.

Departamentos de Enfermedades Respiratorias ${ }^{1}$ y Reumatología ${ }^{2}$, Pontificia Universidad Católica de Chile, Santiago, Chile.

aAlumna $7^{0}$ Año de Medicina, Universidad de los Andes

Correspondencia a: Dr. Fernando Saldías Peñafiel. Departamento de Enfermedades Respiratorias, Pontificia Universidad Católica de Chile. Marcoleta 352 - $1^{\text {er }}$ Piso, Santiago, Chile. Teléfonos: 6331541 - 3543242. Fax: 6335255.

Email: fsaldias@med.puc.cl 
$\mathrm{L}$ a polimiositis y dermatomiositis (PM/DM) son enfermedades inflamatorias del tejido conectivo de etiología desconocida que afectan principalmente los músculos esqueléticos, piel y otros órganos internos ${ }^{1}$. El compromiso pulmonar y las complicaciones respiratorias son frecuentes en la evolución de la enfermedad, entre las cuales se incluyen la debilidad de los músculos respiratorios, neumonía aspirativa, enfermedad pulmonar intersticial, infecciones y daño pulmonar por drogas $^{2-4}$. Las enfermedades pulmonares intersticiales afectan a $23,1 \%-65 \%$ de los pacientes con $\mathrm{PM} / \mathrm{DM}$, siendo los principales patrones histológicos la neumonía intersticial inespecífica (NSIP), neumonía intersticial usual (UIP), neumonía en organización (COP) y daño alveolar difuso (DAD). Los esteroides e inmunosupresores son el tratamiento habitual de los cuadros pulmonares asociados a esta entidad ${ }^{2,3}$.

La PM/DM son enfermedades autoinmunes esporádicas, la respuesta clínica con el tratamiento inmunosupresor es parcial en dos tercios de los casos, y tienen tasas de letalidad elevadas que fluctúan entre $23 \%$ y $73 \%$ a cinco años ${ }^{1,3,5}$. Los principales factores de mal pronósticos identificados son la edad avanzada, retardo en el diagnóstico e inicio de tratamiento, la presencia de neoplasia, el compromiso

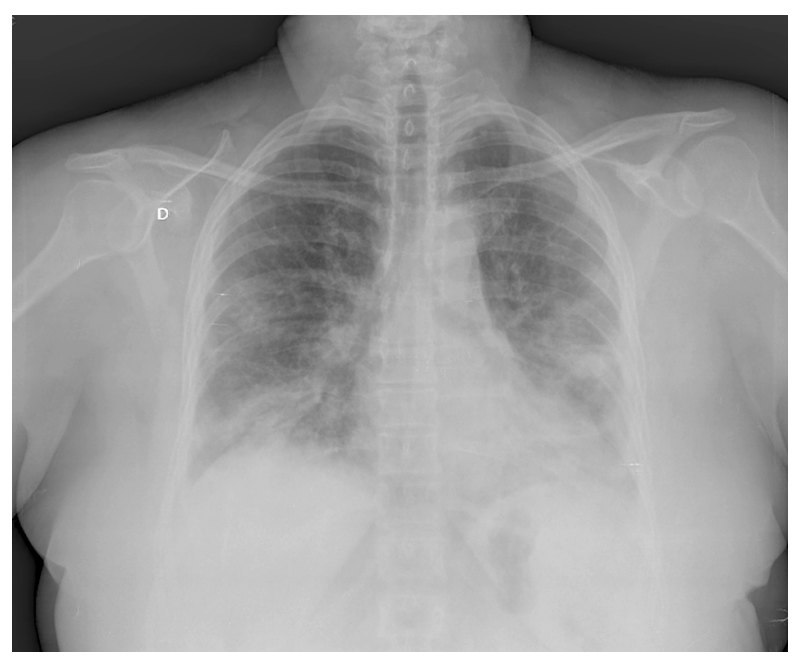

Figura 1. La radiografía de tórax en el momento del diagnóstico muestra opacidades intersticiales y de relleno alveolar bilaterales de predominio en ambos lóbulos inferiores (mayo de 2005). pulmonar intersticial y los patrones histológicos variedad UIP y DAD.

La neumonía en organización (COP) es un síndrome clínico-patológico de causa desconocida, descrito por Epler en 1985, que se caracteriza por la presencia de focos de tejido conectivo laxo en la vía aérea distal asociado a inflamación crónica alveolar ${ }^{6,7}$. Esta entidad clínica puede ser de origen desconocido o criptogénica, o asociada a infecciones, fármacos, neoplasias hematológicas, enfermedad inflamatoria intestinal y enfermedades del tejido conectivo ${ }^{8}$. Se presenta el caso clínico de una paciente con dermatomiositis que consultó por síntomas respiratorios y compromiso pulmonar intersticial alrededor de un año antes que aparecieran las manifestaciones sistémicas de la enfermedad, quien no respondió al tratamiento inicial con esteroides y otros agentes inmunosupresores, por lo cual fue manejada con rituximab obteniendo buena respuesta clínica.

\section{CASO CLÍNICO}

Mujer de 55 años, ex-fumadora de 10 paq/año, portadora de diabetes mellitus tipo 2 e hipertensión arterial crónica en tratamiento con régimen, metformina, glibenclamida y atenolol, consultó en mayo de 2005 por disnea de esfuerzo progresiva de un mes de evolución asociado a tos seca. No refería compromiso del estado general, baja de peso, fiebre, calofríos, dolor torácico, artralgias, fotosensibilidad, ni lesiones cutáneas. No tenía exposición a aves ni sustancias tóxicas y no había realizado viajes.

En el examen físico destacaba paciente en buenas condiciones generales, obesa, IMC: 31 $\mathrm{kg} / \mathrm{m}^{2}$, FC: 78 lat/min, PA: 135/90 mmHg, FR: $20 \mathrm{resp} / \mathrm{min}, \mathrm{T}: 36^{\circ} \mathrm{C}$. El examen cardiaco era normal y en el examen pulmonar se auscultaban crepitaciones en ambas bases pulmonares. No tenía adenopatías, debilidad muscular, hipocratismo digital, artritis, ni lesiones cutáneas u oculares.

En la radiografía de tórax se observaban múltiples opacidades de relleno alveolar, especialmente en los lóbulos inferiores (Figura 1) y el estudio funcional respiratorio mostraba una limitación espirométrica restrictiva y capacidad de difusión de monóxido de carbono 
(DLCO) disminuida, con fuerza muscular conservada. La tomografía computada de tórax mostraba múltiples focos de vidrio esmerilado de distribución periférica y subpleural, especialmente en lóbulos inferiores (Figura 2). Los exámenes de laboratorio general (hemograma, pruebas de función renal y hepática, perfil lipídico, sedimento de orina) eran normales, y los exámenes inmunológi$\cos$ (anticuerpos antinucleares y antiADN, factor reumatoideo, anticuerpos anticitoplasma de neutrófilos) eran negativos.

En junio de 2005 se realizó biopsia pulmonar por videotoracoscopia que fue compatible con el diagnóstico de neumonía en organización (Figura 3), se planteó el diagnóstico de COP primario y se inició tratamiento con prednisona $1 \mathrm{mg} / \mathrm{kg} /$ día. La paciente evolucionó favorablemente con disminución de la disnea y mejoría parcial del compromiso funcional respiratorio, reduciéndose progresivamente las dosis de esteroides en el curso de un año. En la Tabla 1 se describe la evolución de las pruebas funcionales respiratorias.

En junio de 2006, al descender la dosis de prednisona a $10 \mathrm{mg}$ diarios, la paciente refirió sensación febril, mialgias, artralgias, debilidad muscular proximal y luego la aparición de rash heliotropo, pápulas de Gottron y eritema periun-

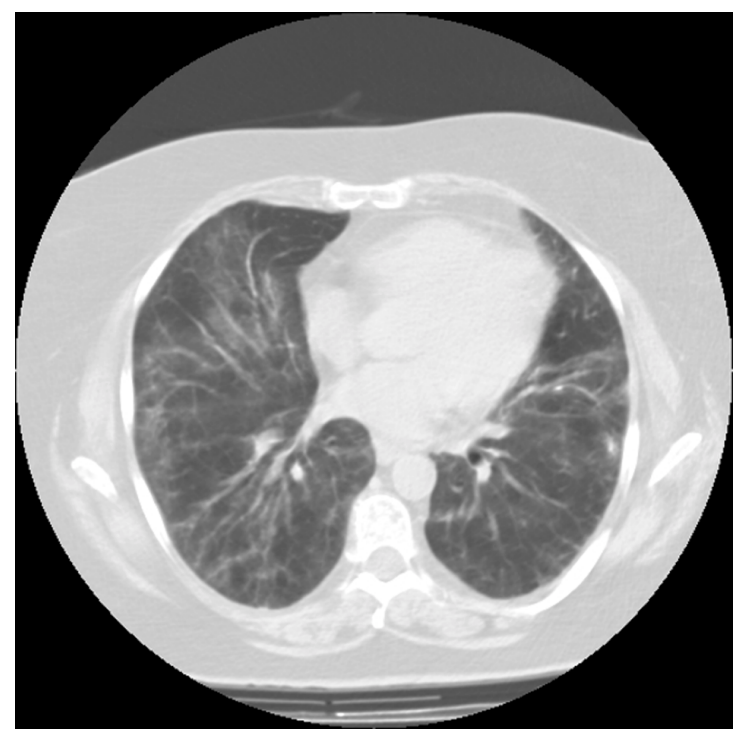

Figura 2. La tomografía computarizada de tórax en el momento del diagnóstico muestra opacidades reticulares y en vidrio esmerilado bilaterales de predominio en ambos lóbulos inferiores (junio de 2005). gueal en las manos, asociado a aumento de la disnea, deterioro funcional respiratorio y progresión de las opacidades reticulares y en vidrio esmerilado en la tomografía de tórax. Se efectuó fibrobroncoscopia con lavado broncoalveolar para descartar infección oportunista, aumentando la dosis de prednisona a $1 \mathrm{mg} / \mathrm{kg} /$ día por sospecha de reactivación de COP. En los exámenes solicitados destacan los anticuerpos anti Ro y anti Jo-1 positivos, elevación de enzimas musculares (creatinfosfokinasa: $2.910 \mathrm{U} / \mathrm{L}, \mathrm{LDH}: 512 \mathrm{U} / \mathrm{L}$ ) y la electromiografía demostró un patrón miopático (potenciales de baja amplitud, corta duración y reclutamiento aumentado y actividad espontánea compatible con denervación aguda: fibrilaciones y ondas positivas). Evaluado en conjunto con el equipo de reumatología se plantea el diagnóstico de dermatomiositis con compromiso pulmonar intersticial agregándose a la terapia infusión de inmunoglobulinas $400 \mathrm{mg} / \mathrm{kg} /$ día durante cinco días, pulsos de ciclofosfamida endovenosa y luego azatioprina $100 \mathrm{mg} /$ día.

La paciente evolucionó con deterioro progresivo de la capacidad funcional, fiebre vespertina, mialgias, artralgias, debilidad muscular proximal, deterioro de la capacidad vital, DLCO y prueba de caminata en seis minutos (enero 2007). La falta de respuesta clínica y

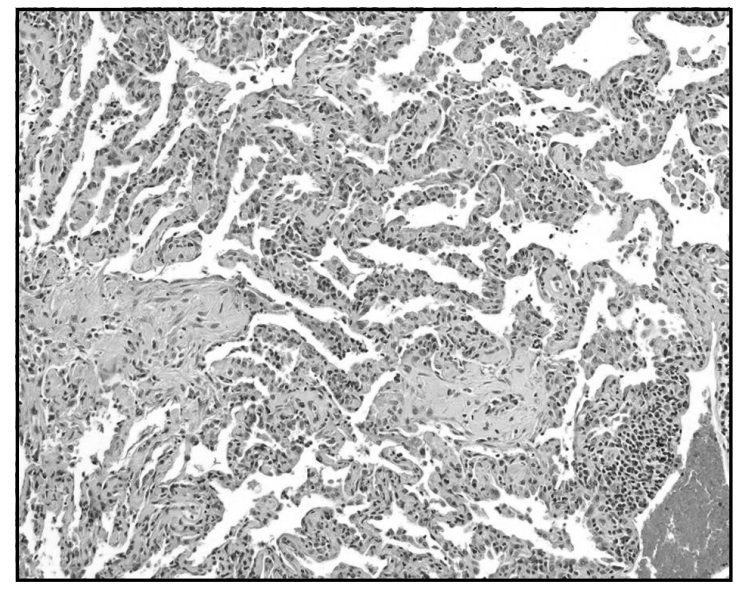

Figura 3. Muestra histológica obtenida mediante biopsia pulmonar por VATS, que muestra los tabiques engrosados, con edema e infiltrados de células linfoides, de distribución irregular; los neumocitos aparecen hiperplásicos y se ven los espacios aéreos ocupados por masas polipoides de aspecto mixoide. (Hematoxilina eosina $100 \mathrm{X}$ ). 
Tabla 1. Evaluación funcional respiratoria de paciente con dermatomiositis refractaria y enfermedad pulmonar difusa tratada con rituximab

\begin{tabular}{|c|c|c|c|c|c|c|}
\hline Evolución & Mayo-05 & Enero-06 & Julio-06 & Enero-07 & Julio-07 & Abril-08 \\
\hline Tratamiento & PRED & PRED & PRED-C & PRED-AZ & PRED-AZ-R & PRED-AZ-R \\
\hline \multicolumn{7}{|l|}{ Espirometría } \\
\hline CVF & 2,05 (71\%) & 2,03 (70\%) & 2,39 (83\%) & 1,82 (63\%) & 1,89 (65\%) & 2,35 (80\%) \\
\hline $\mathrm{VEF}_{1}$ & 1,66 (69\%) & 1,68 (70\%) & 2,02 (85\%) & 1,55 (65\%) & 1,56 (65\%) & 2,00 (84\%) \\
\hline $\mathrm{VEF}_{1} / \mathrm{CVF}$ & $81 \%$ & $83 \%$ & $85 \%$ & $85 \%$ & $83 \%$ & $85 \%$ \\
\hline \multicolumn{7}{|l|}{ DLCO } \\
\hline DLCO & 12,7 (53\%) & $10,8(47 \%)$ & 13,0 (55\%) & 8,4 (37\%) & $9,2(40 \%)$ & 9,5 (43\%) \\
\hline VA & 3,32 (53\%) & 2,72 (46\%) & 3,54 (56\%) & 2,3 (38\%) & 2,5 (41\%) & $3,0(50 \%)$ \\
\hline DLCO/VA & 3,81(102\%) & 3,96 (102\%) & 3,61 (96\%) & 3,7 (97\%) & 3,7 (98\%) & 3,2 (86\%) \\
\hline \multicolumn{7}{|l|}{ DR6M } \\
\hline Distancia & 420 (86\%) & 443 (92\%) & 434 (90\%) & 426 (88\%) & 420 (86\%) & 445 (92\%) \\
\hline $\mathrm{SaO}_{2} \mathrm{R}-\mathrm{E}$ & $90 \%-83 \%$ & $95 \%-86 \%$ & $94 \%-93 \%$ & $95 \%-75 \%$ & $96 \%-80 \%$ & $95 \%-85 \%$ \\
\hline FC R - E & $87-110 x^{\prime}$ & $85-126 x^{\prime}$ & $69-122 x^{\prime}$ & $74-120 x^{\prime}$ & 73-102 x' & 78-112 x' \\
\hline Disnea R - E & $1-9$ & $1-6$ & $0-3$ & $0-9$ & $1-4$ & $0-3$ \\
\hline
\end{tabular}

Nota: CVF: capacidad vital forzada, $\mathrm{VEF}_{1}$ : volumen espiratorio forzado de $1^{\mathrm{er}}$ segundo, DLCO: capacidad de difusión de CO, VA: volumen alveolar, DR6M: Prueba de caminata de 6 min, $\mathrm{SaO}_{2}$ : saturación arterial de oxígeno, FC: frecuencia cardiaca, R: reposo, E: ejercicio. PRED: Prednisona, C: Ciclofosfamida, AZ: Azatioprina, R: Rituximab. Los valores son expresados como valor absoluto y porcentaje del valor teórico.

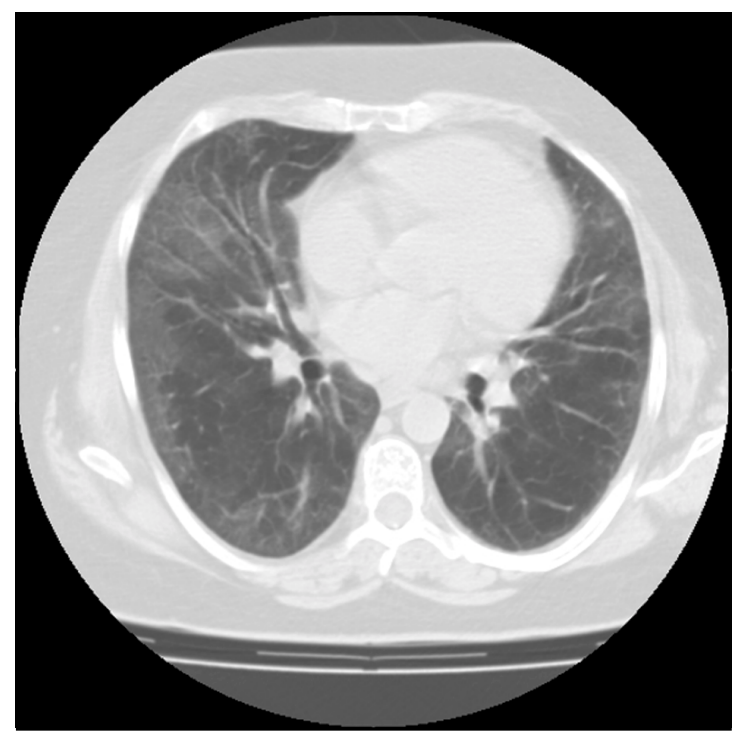

Figura 4. La tomografía computarizada de tórax muestra regresión parcial de las opacidades reticulares y en vidrio esmerilado seis meses después del tratamiento con rituximab (noviembre de 2007). progresión del compromiso respiratorio nos obligó a buscar terapias alternativas, decidiéndose administrar dos dosis de rituximab de $1 \mathrm{~g}$ cada 14 días, con lo cual se evidenció mejonía significativa de los parámetros clínicos y funcionales antes mencionados a los dos meses de recibir el tratamiento (julio 2007). A los seis meses, reaparecieron los síntomas sistémicos, fiebre vespertina, artralgias, mialgias y debilidad muscular proximal, indicándose nuevo ciclo de rituximab en noviembre de 2007 ( $1 \mathrm{~g}$ por 2 veces); con lo que se logró estabilizar su condición clínica, los exámenes de función pulmonar han mejorado en forma significativa en los seis meses de seguimiento (abril 2008) y la tomografía de tórax demostró importante regresión de las opacidades reticulares y de vidnio esmerilado de los lóbulos inferiores (Figura 4).

\section{DisCUSIÓN}

La neumonía en organización criptogénica es una variedad de neumonía intersticial idiopática carac- 
terizada por la presencia de focos de relleno alveolar que pueden estar asociados a bronquiolitis ${ }^{8,9}$, puede ser idiopática o criptogénica y secundaria a enfermedades colágeno vasculares, infecciones, neoplasias hematológicas, enfermedades inflamatorias intestinales y fármacos ${ }^{8-10}$.

El compromiso pulmonar en la PM/DM incluye la debilidad de los músculos respiratorios, riesgo de neumonía aspirativa, infección por gérmenes oportunistas, toxicidad pulmonar por drogas y el compromiso intersticial-4,11-13. La enfermedad pulmonar intersticial se presenta en $20 \%$ a $60 \%$ de los pacientes con PM/DM, puede preceder a las manifestaciones sistémicas hasta en $25 \%$ de los enfermos, con un tiempo promedio que varía entre 100 y 354 días ${ }^{12-14}$. Las principales formas histológicas son la neumonía intersticial usual y neumonía inespecífica, siendo menos frecuentes la neumonía en organización y daño alveolar difuso ${ }^{3,12,13}$. En nuestra paciente, el compromiso pulmonar precedió a las manifestaciones sistémicas de la enfermedad en alrededor de un año y el diagnóstico histológico fue compatible con una neumonía en organización.

El compromiso pulmonar suele acontecer en la edad media de la vida, sin diferencias por género, se manifiesta por disnea de esfuerzo de evolución subaguda y tos seca asociada a fiebre y compromiso del estado general en la mitad de los casos ${ }^{2-}$ 4. En el examen pulmonar se auscultan crepitaciones, siendo infrecuente la presencia de hipocratismo digital $(<5 \%)$. No se ha demostrado asociación con el tabaquismo.

Los exámenes de laboratorio pueden mostrar aumento de los marcadores de la inflamación en 70\%-80\% de los casos (leucocitosis, proteína C reactiva y eritrosedimentación elevadas) ${ }^{3,4,11}$. Los pacientes suelen presentar anticuerpos anti Ro y antisintetasa elevados, como ocurrió en nuestra paciente; de hecho, los anticuerpos anti Jo-1 se consideran un marcador específico de compromiso pulmonar en la $\mathrm{PM} / \mathrm{DM}^{4,11,14}$.

En los estudios de función pulmonar destaca la alteración espirométrica de tipo restrictiva, asociada a disminución de la DLCO y desaturación arterial en ejercicio ${ }^{11-14}$. El estudio imagenológico muestra opacidades reticulares y en vidrio esmerilado difusas, de predominio en lóbulos inferiores ${ }^{11-14}$. El patrón histológico observado corresponde principalmente a NSIP (65\%-81\%), UIP (9\%), COP (5\%) y DAD (5\%), aunque rara vez se efectúa biopsia pulmonar quirúrgica cuando se tiene el diagnóstico de la enfermedad colágenovascular 3,4,11-14.

La sobrevida de los pacientes con PM/DM y compromiso pulmonar intersticial a tres y cinco años es alrededor de $74 \%$ y $50 \%$, respectivamente $\mathrm{t}^{1,3,11,12}$, dentro de los factores de mal pronóstico se reconoce la dermatomiositis amiopática, los patrones histológicos de neumonía intersticial usual y daño alveolar difuso, la capacidad vital menor de $60 \%$ y DLCO menor de $45 \%$ en el momento del diagnóstico $11,12,14$.

El tratamiento de elección son los corticoides orales en dosis elevadas, obteniendo respuesta favorable en $50 \%$ de los $\operatorname{casos}^{11-14}$. Entre los factores que predicen una buena respuesta al tratamiento esteroidal se encuentran los pacientes jóvenes, diagnóstico de polimiositis, patrón histológico de COP, enzimas musculares elevadas y patrón radiológico con predominio de vidrio esmerilado.

En los pacientes con progresión de la enfermedad a pesar de la terapia esteroidal, lo cual ocurre en un tercio de los enfermos, caracterizado por aumento de la disnea, deterioro de la función pulmonar o progresión de las imágenes radiológicas, se recomienda adicionar ciclofosfamida, ciclosporina A o azatioprina, habiendo descartado previamente una infección pulmonar por gérmenes oportunistas ${ }^{11,14,15}$.

En los pacientes con pobre respuesta a la terapia inmunosupresora, con síntomas sistémicos y progresión del compromiso pulmonar, como ocurrió en nuestra paciente, se puede agregar inmunoglobulinas endovenosas en dosis inmunorreguladoras o rituximab ${ }^{15-17}$. El rituximab es un anticuerpo monoclonal quimérico dirigido contra el antígeno $\mathrm{CD}_{20}$ de las células $\mathrm{B}$ maduras ${ }^{16-18}$. Este antígeno está ampliamente distribuido en la superficie de estas células regulando su función y activación ${ }^{19}$. El rituximab produce depleción de linfocitos $\mathrm{B}$ a través de diversos mecanismos como inducción de apoptosis, lisis mediada por complemento, inhibición de crecimiento y citotoxicidad a través de la fracción $\mathrm{Fc}^{20}$. Luego de la administración parenteral de rituximab en dosis de $125-375 \mathrm{mg} / \mathrm{m}^{2}$ de superficie corporal, es posible detectar el anticuerpo en sangre durante 3 a 6 semanas. El recuento de células $B$ 
periféricas disminuye por debajo del valor normal después de la primera dosis, para luego de seis meses recuperar los valores normales $y$, finalmente, normalizarse después de 9 a 12 meses de terminado el tratamiento. Existen reportes de casos y pequeñas series de pacientes que han examinado su empleo en diversas mesenquimopatías refractarias a corticoides y otros inmunosupresores obteniendo respuesta clínica favorable ${ }^{16-19}$. El perfil de seguridad y tolerancia del fármaco es adecuado, obteniendo respuesta clínica a corto plazo en dos tercios de

\section{REFERENCIAS}

1. Marie I, Hachulla E, Hatron Py, Hellot MF, Levesoue H, Devulder B et AL. Polymyositis and dermatomyositis: short term and longterm outcome, and predictive factors of prognosis. J Rheumatol 2001; 28: 2230-7.

2. DiCKey BF, MYERS AR. Pulmonary disease in polymyositis/dermatomyositis. Semin Arthritis Rheum 1984; 14: $60-76$.

3. Tazelaar HD, Viggiano RW, Pickersgill J, Colby TV. Interstitial lung disease in polymyositis and dermatomyositis. Clinical features and prognosis as correlated with histologic findings. Am Rev Respir Dis 1990; 141: 727-33.

4. Marie I, Hatron PY, Hachula E, Wallaert B, MichonPasturel U, Devulder B. Pulmonary involvement in polymyositis and in dermatomyositis. J Rheumatol 1998; 25: 1336-43.

5. Airio A, Kautiainen H, Hakala M. Prognosis and mortality of polymyositis and dermatomyositis patients. Clin Rheumatol 2006; 25: 234-9.

6. Epler GR, Colby TV, McLoud TC, Carrington CB, GAENSLER EA. Bronchiolitis obliterans organizing pneumonia. N Engl J Med 1985; 312: 152-8.

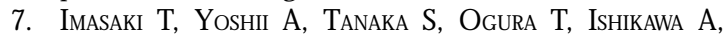
TAKAHASHI T. Polymyositis and Sjögren's Syndrome associated with bronchiolitis obliterans organizing pneumonia. Intern Med 1996; 35: 231-5.

8. American Thoracic Society/European Respiratory Society International Multidisciplinary Consensus Classification of the Idiopathic Interstitial Pneumonias. Am J Respir Crit Care Med 2002; 165: 277-304.

9. KING TE JR. Clinical advances in the diagnosis and therapy of the interstitial lung diseases. Am J Respir Crit Care Med 2005; 172: 268-79.

10. CORDIER JF. Cryptogenic organising pneumonia. Eur Respir J 2006; 28: 422-46. los enfermos y reaparición de los síntomas en promedio a los 6-8 meses ${ }^{17-19}$.

Nuestro objetivo es entregar un mensaje de alerta a los clínicos frente a pacientes con neumonía en organización (COP) de origen desconocido, en relación a etiología secundaria tanto en el momento del diagnóstico como durante la evolución de la enfermedad, así como describir nuestra primera experiencia en el tratamiento de la dermatomiositis con compromiso pulmonar refractaria a corticoides con rituximab.

11. Douglas WW, Tazelaar HD, Hartman TE, Hartman RP, Decker PA, Schroeder DR et aL. Polymyositis-dermatomyositis-associated interstitial lung disease. Am J Respir Crit Care Med 2001; 164: 1182-5.

12. Cottin V, Thivolet-Béjui F, Reynaud-Gaubert M, Cadranel J, Delaval P, Ternamian PJ et al. Interstitial lung disease in amyopathic dermatomyositis, dermatomyositis and polymyositis. Eur Respir J 2003; 22: 24550.

13. Kang EH, Lee EB, Shin KC, Im CH, Chung DH, HaN SK, Song YW. Interstitial lung disease in patients with polymyositis, dermatomyositis and amyopathic dermatomyositis. Rheumatology 2005; 44: 1282-6.

14. Marie I, Hachula E, Chérin P, Dominique S, Hatron PY, НеLOT MF ET AL. Interstitial lung disease in polymyositis and dermatomyositis. Arthritis Rheum 2002; 47: 614-22.

15. YTTERBERG SR. Treatment of refractory polymyositis and dermatomyositis. Curr Rheumatol Rep 2006; 8: 167-73.

16. Gottenberg J, Guilevin L, Lambotte O, Combe B, Aluanore Y, Cantagrel A et al. Tolerance and short term efficacy of rituximab in 43 patients with systemic autoimmune diseases. Ann Rheum Dis 2005; 64: 913-20.

17. Mok CC, Ho LY, To CH. Rituximab for refractory polymyositis: an open-label prospective study. J Rheumatol 2007; 34: 1864-8.

18. Brulhart L, Waldburger JM, Gabay C. Rituximab in the treatment of antisynthetase syndrome. Ann Rheum Dis 2006; 65: 974-5.

19. LeVINE TD. Rituximab in the treatment of dermatomyositis: an open-label pilot study. Arthritis Rheum 2005; 52: 601-7.

20. Cragg MS, Walshe CA, Ivanov AO, Giennie MJ. The biology of CD20 and its potential as a target for mAb therapy. Curr Dir Autoimmun 2005; 8: 140-74. 